Meta

Journal des traducteurs

Translators' Journal

\title{
Peut-on envisager l'avenir de la traduction sans plaisir ? Pour une érotique du traduire
}

\section{Sathya Rao}

Volume 50, numéro 4, décembre 2005

Pour une traductologie proactive - Actes

For a Proactive Translatology — Proceedings

Por una traductología proactiva - Actas

URI : https://id.erudit.org/iderudit/019855ar

DOI : https://doi.org/10.7202/019855ar

Aller au sommaire du numéro

Éditeur(s)

Les Presses de l'Université de Montréal

ISSN

0026-0452 (imprimé)

1492-1421 (numérique)

Découvrir la revue

Citer cet article

Rao, S. (2005). Peut-on envisager l'avenir de la traduction sans plaisir ? Pour une érotique du traduire. Meta, 50(4). https://doi.org/10.7202/019855ar
Résumé de l'article

Si la traduction a donné lieu à une éthique (Berman) et à une politique (Venuti), elle n'a jamais pu réellement se penser comme érotique. Plus exactement, lorsqu'elle s'y est essayée, la traductologie s'est systématiquement fourvoyée, prenant tantôt le parti du traducteur viril violentant le texte-source, tantôt celui du traducteur docile se pliant à l'Original.

La force de subversion d'une érotique de la traduction consiste à prendre la mesure de la sensualité de l'acte de traduire. Si le rapport au corps textuel de l'autre n'est jamais aussi aseptisé que le suppose la théorie, de même il n'est pas non plus aussi terre-à-terre que l'admet la pratique. Dans la mouvance de Lévinas, il faut envisager la caresse comme un geste paradigmatique effleurant le corps du texte, mais dont la visée n’est ni de le posséder ni de le trahir.
Ce document est protégé par la loi sur le droit d'auteur. L'utilisation des services d’Érudit (y compris la reproduction) est assujettie à sa politique d'utilisation que vous pouvez consulter en ligne.

https://apropos.erudit.org/fr/usagers/politique-dutilisation/ 


\title{
Peut-on envisager l'avenir de la traduction sans plaisir? Pour une érotique du traduire
}

\author{
SATHYA RAO \\ Université Concordia, Université de Montréal, Montréal, Canada \\ sathya_rao6@hotmail.com
}

\section{RÉSUMÉ}

Si la traduction a donné lieu à une éthique (Berman) et à une politique (Venuti), elle n'a jamais pu réellement se penser comme érotique. Plus exactement, lorsqu'elle s'y est essayée, la traductologie s'est systématiquement fourvoyée, prenant tantôt le parti du traducteur viril violentant le texte-source, tantôt celui du traducteur docile se pliant à l'Original.

La force de subversion d'une érotique de la traduction consiste à prendre la mesure de la sensualité de l'acte de traduire. Si le rapport au corps textuel de l'autre n'est jamais aussi aseptisé que le suppose la théorie, de même il n'est pas non plus aussi terre-à-terre que l'admet la pratique. Dans la mouvance de Lévinas, il faut envisager la caresse comme un geste paradigmatique effleurant le corps du texte, mais dont la visée n'est ni de le posséder ni de le trahir.

\begin{abstract}
Envisioned in ethical (Berman) and political terms (Venuti), translation was never successfully considered in erotic terms. Indeed, when translatologists attempted to do so, they emphasized either the viewpoint of the translator brutalizing the source-text or that of the translator subjugated to the Original.

The subversive power of an "erotics of translation" consists in unleashing translation's sensual activity. The relationship to the textual body is never as sterile as theoreticians posit or as crude as practitioners believe. Following Levinas, the notion of caress could be considered as a paradigmatic gesture touching lightly the textual body, a gesture whose aim is neither to possess this body nor to betray it.
\end{abstract}

\section{MOTS-CLÉS/KEYWORDS}

amour, érotique de la traduction, pornographie, corps, visibilité

\section{L'amour en traduction}

Il peut sembler incongru, si ce n'est choquant, de voir la traduction associée aux motifs de l'érotique et de la pornographie. Pourtant, l'amour au sens large est une composante essentielle de la pensée traductologique ; elle s'y manifeste sous formes diverses qui ne revendiquent pas nécessairement le label d'érotique. Interrogez un traducteur sur les raisons profondes de son acte, il vous répondra qu'il traduit par amour de sa propre langue ou de la langue de l'autre, par désir de faire partager une oeuvre chère ou bien tout simplement pour le plaisir de traduire.

Nombre de traductologues contemporains, à commencer par V. Larbaud et A. Berman, ont fait la part belle à l'amour en traduction. Pour le premier, l'œuvre de traduction procède d'un « instinct primitif d'appropriation » (Larbaud 1946 : 23-24) dont la domestication conduit, dans le meilleur des cas, à un subtil jeu de séduction où « délicatesse » et « virilité » doivent opérer de concert. Pour le second, la vocation du traducteur tient à une mystérieuse «pulsion de traduction » qui trouve son fondement autant dans la psychanalyse freudienne que dans le vitalisme romantique. Ainsi, la pulsion de traduction ramène-t-elle l'activité traduisante au cœur du sujet, appelant dès lors une psychanalyse du traducteur dont Berman n'a jamais pu poser les bases. Oeuvrant donc dans l'intériorité du sujet traducteur, la pulsion de traduction est le site d'une double sublimation qui détermine la visée soit « métaphysique », soit « éthique » du geste traductif (Berman $1984: 23$ ).

Sur un registre un peu différent, comment ne pas évoquer l'analyse que fait A. Berman du poème de John Donne « Going to bed » dans Pour une critique des traductions : John 
Donne? Choix littéraire aussi peu anodin que celui que fait G. Steiner en ouverture de son monumental After Babel. De «Going to bed » au deuxième acte de Cymbeline en passant par la sublime retraduction du Cantique des cantiques par $\mathrm{H}$. Meschonnic se fait jour un intérêt de traduction commun pour l'amour et sa prose. Intérêt que la pensée traductologique n'a, semble$\mathrm{t}$-il, jamais pris au sérieux. Et si les textes érotiques avaient quelque chose de « paradigmatique » au sens où J-R. Ladmiral l'affirme à propos des textes philosophiques ? Il y aurait à entreprendre la généalogie de l'oubli (ou du masquage pudibond) de l'amour en traduction...

Si l'amour au sens large peut être cause ou visée de traduction, il peut également se donner à voir sous les traits de métaphores. Un rapide examen du corpus traductologique suffit à nous convaincre de leur omniprésence : « fidélité », « infidélité », « pénétration », «possession », « soumission», « viol», «séduction », etc. Chargés de connotations idéologiques que le féminisme aura su décoder, ces termes renforcent le parallèle entre relation d'amour et relation de traduction. Parallèle que G. Steiner aura élevé à son niveau le plus général : «Eros et la langue forment une engrenage continu. Rapports sexuels, rapports parlés, copule et copulation sont des sous-catégories de la communication. Ils proviennent du besoin de l'être de sortir de lui-même pour comprendre, au niveau de l'intellect et dans l'espace, un autre être. L'acte d'amour est intensément sémantique. » (Steiner 1998 : 48).

Dans son sens le plus large, l'érotique de la traduction est avant tout la tentative de prendre au sérieux les choses de l'amour en traduction. Dans cette mesure, elle s'oppose à cette pudibonderie traductologique qui consisterait à se voiler la face, à éviter de parler d'amour (ou bien à n'en parler que d'une seule façon) au nom d'une certaine morale. Cette morale, c'est autant celle du littéralisme le plus incarné - qui refuse de faire place au jeu de l'interprétation que celle de la compréhension la plus abstraite - qui néglige l'épaisseur charnelle de la lettre. Aux yeux de l'érotique, nous le verrons, il y a, une (f)rigidité de la lettre comme il y a un exhibitionnisme du sens. Fin observateur de son temps, l'auteur de L'amant de lady Chatterley ne s'y est point trompé :

\footnotetext{
«Ainsi, entre le puritain morne et rassis, toujours menacé de succomber sur le tard à l'indécence sexuelle; entre la personne à la mode de la jeune génération qui dit : « Nous pouvons tout faire ; si nous voulons penser une chose nous pouvons la faire »; et, enfin le barbare à l'âme basse, à l'esprit impur qui recherche la saleté, le champ d'action de ce livre est bien étroitement limité. Mais je leur dis, à tous : « Gardez vos perversions de puritanisme, ou de dévergondage à la mode, ou de simple grossièreté. Quant à moi, je défends mon livre et ma position : la vie n'est acceptable que si l'esprit et le corps vivent en bonne intelligence, s'il y a un naturel équilibre entre eux, et s'ils éprouvent un respect mutuel l'un pour l'autre. » (Lawrence 1993 : 52)
}

Ainsi, du puritanisme à l'obscénité, n'est-il question, à quelques nuances près, que d'une seule et même pornographie et ce, quelques soient les époques. Le propos de D.H. Lawrence n'est pas seulement critique, il ouvre sur la perspective d'une réconciliation qui vaudrait, à bien des égards, pour la traduction elle-même. Aussi, L'amant de lady Chatterley doit-il être lu, mais aussi traduit (pas exclusivement bien sûr) comme une tentative pragmatique de «mettre en harmonie la conscience de nos sensations corporelles avec ces sensations elles-mêmes, la conscience de l'acte avec l'acte lui-même. » (Lawrence 1993 : 50).

Une des tâches critiques de l'érotique consistera justement à examiner autant ce que les érotiques traductologiques ${ }^{1}$ ont d'excessif que l'extrême sophistication de leurs tendances sadomasochistes. On pourra, par exemple, s'interroger sur le type d'érotique à l'œuvre dans les Belles Infidèles et méditer sur la position paradoxale du traducteur violentant le texte-source au nom d'un amour immodéré pour sa langue maternelle. La rigueur analytique de l'érotique imposerait également de faire l'examen de ces coups et blessures linguistiques portés au corps du texte-source. A. Berman n'a pas manqué de le souligner : l'amour en traduction, c'est également la haine de la langue de l'autre (ou bien de sa propre langue).

En matière d'érotique, la traductologie féministe aurait pu faire office de précurseur si elle n'avait pas cédé le pas, devant l'urgence de la situation, à la revendication politique. Le propos de l'érotique du traduire, au sens où nous la concevons, est moins de replacer la femme ou l'homme au centre de la traduction ou de la langue que de repenser leur relation. En effet, la fonction pragmatique de la caresse n'a pas besoin que les polarités sexuelles soient clairement différenciées. Plus exactement, ces polarités ne doivent pas être tenues (idéologiquement) pour acquises, mais chaque fois réinventées à l'horizon du rapport (de traduction) lui-même. 
Dans un sens que l'on dira plus engagé, l'érotique de la traduction non seulement fait œuvre de résistance, mais porte un projet d'amour pour la traduction. Ce projet se veut une alternative au modèle « pornologique » du traduire tel qu'il est promu par la globalisation (Rao 2005). Les chiffres le prouvent : il existe une étroite corrélation entre l'avènement de l'économie globale et la prolifération de l'industrie pornographique. Derrière les statistiques se cache une réalité bien plus subtile, celle de la généralisation d'une certaine technologie de l'amour (en traduction). Nous poserons, en des termes proches de ceux de J. Baudrillard, que le propre de cette technologie est d'idéaliser le corps (textuel) au point de lui faire perdre toute sensibilité. Ainsi, élevé à l'état d'image ou de concept, le corps (textuel) finit par perdre toute profondeur, toute intériorité. C'est l'anesthésie généralisée de la chair. En bref, l'excès de sens tue le sens, insensibilise en abstrayant le corps dans l'universel du sens. De la pin-up sur papier glacé au corps surexposé du texte-source, il y va de la même logique, à savoir la production d'une illusion de visibilité absolue, de traductibilité intégrale. Illusion dont une des manifestations est l'exigence de fluency pointée du doigt par L. Venuti. Alors qu'il oeuvre, avec plus ou moins de conscience, à un projet global d'insensibilisation du corps (textuel), le traducteur perd de vue (ou réinvente dans le meilleur des cas ?) sa profondeur.

Multipliant les images de lui-même, le traducteur aura, par la force des choses, de moins en moins à cacher : il s'engage en théorie, part en lutte contre son invisibilité notoire, s'interroge sur sa responsabilité, affirme avec force ses choix (politiques), donne ses yeux à la science ${ }^{2}$, en bref assume plus que jamais son arbitraire. Ce faisant, plus le traducteur s'affiche, plus il prend le risque de perdre en sensibilité. Si, comme beaucoup l'affirment, le traducteur pâtit d'une position d'invisibilité relative, il ne faudrait pas non plus minimiser le risque de son excès de visibilité. Le danger est donc double, c'est autant celui de l'exploitation, voire de la prostitution du traducteur que celui de sa «starification ». En ce sens, il y a autant à lutter pour l'amélioration du statut politico-juridique du traducteur que pour le maintien - sous des conditions renouvelées - de son propre «mystère », de son invisibilité. Notre propos ici n'est ni de regretter l'époque bénite où le traducteur savait se tenir, encore moins d'entonner la louange sourcière de sa discrétion légendaire. Nous disons simplement qu'à force d'être pris (ou médiatisé) dans les rets du discours mondains, le traducteur (ou la traduction) risque de ne plus avoir le recul suffisant pour pouvoir intervenir dans le monde. Face au danger de la « transparence » ou de nudité intégrale du traducteur, il convient de poser la question éminemment érotique de son silence. Au sens où l'entend l'érotique, s'engager - c'est-à-dire assumer le risque d'aimer, ce n'est donc pas tant prendre part aux bruits et à la fureur du monde que de savoir s'y rendre invisible afin d'entreprendre une action plus tangible. De cela, on pourra sans doute conclure que l'érotique de la traduction ne va pas sans une certaine économie de moyens, pour ne pas dire une modestie.

Un deuxième trait caractéristique de la sémiotique pornologique est l'instauration d'une économie capitaliste du corps textuel. Reposant sur un principe de ségrégation, cette économie postule des zones de sens capitalisant à elles seules la totalité du plaisir. Ces zones érogènes valent alors par métonymie pour l'ensemble du corps textuel. Dans cette optique, traduire se résume à trouver les équivalents des noms de ces zones érogènes.

Perdant inévitablement en ductilité, le corps textuel réfléchit le passage métaphoriquemétonymique entre l'absolu du corps-signe (dépourvu, comme tel, d'intériorité et d'extériorité) et la topologie éclatée des zones érogènes. Du point de vue d'une linguistique érotique des corps textuels à venir, il y aurait à faire la critique érotique du nom dans sa prétention pornographique à saisir totalement la chose, à circonscrire absolument le plaisir. En toute rigueur, il faudrait également revenir sur la prétention de la chose à excéder sa nomination (du fait brut de son incomparable réalité), à faire jouir par son manque. En définitive, il n'y a pas de pornologue qui ne soit un nominaliste doublé d'un réaliste ; adaptons : un cibliste doublé d'un sourcier. À la suite des réflexions de R. Barthes sur la lecture, l'érotique souhaiterait interroger, pour sa part, le « plaisir de traduire » en évitant le double registre du métalangage et du réalisme faussement scientifique.

En outre, toute amoureuse qu'elle est, l'érotique n'est pas dupe des variations culturelles et sociales touchant sa propre pratique. Elle n'ignore pas non plus les logiques de pouvoir à l'origine du reclassement symbolique du pornographique en érotique ou inversement ${ }^{3}$. Il est d'usage d'accorder à la littérature érotique un prestige tout « platonicien » qui lui vaudrait un lectorat de qualité, mais aussi un traducteur - généralement universitaire - à la réputation sans tâche. Élevés par M. Heine, G. Bataille et R. Barthes au rang d'œuvre érotique, les romans de 
Sade ont fini par voir leur force subversive s'étioler en tombant dans le domaine public. Que dire encore des traductions esthétisantes de D.H. Lawrence et d'H. Miller ${ }^{4}$ ? S'agissant du rapport entre genres de littérature et classes, le constat empirique de N. Huston est suffisamment explicite : «[...] Tandis que ce l'on a l'habitude d'appeler « érotisme» est consommé surtout par l'élite intellectuelle et professionnelle (hommes et femmes confondus), le roman pornographique «sans mérite littéraire » et le roman à l'eau de rose sont destinés, respectivement, aux hommes et aux femmes des classes populaires. » (Huston 1982 : 29).

\section{L'essence non-platonique de la traduction ou la traduction-caresse}

L'érotique du traduire n'est ni une théorie ni une pratique de l'amour en traduction. De la théorie, l'érotique refuse le platonisme, c'est-à-dire la propension à confondre spéculation et amour de la traduction. Lorsqu'il ne se complaira pas dans l'amour de l'Esprit (ou l'esprit de l'Amour) ${ }^{5}$, le platonicien puritain éprouvera une haine profonde à l'endroit du corps (de la lettre), hyper-sexualisé pour l'occasion. De la pratique, l'érotique rejette la grossièreté, c'est-àdire la certitude empirique (qui ne l'est jamais totalement) qu'aimer la traduction revient à la « sentir dans ses tripes ». Se voyant ramené au plus près du corps du traducteur, le phénomène de la traduction acquiert une telle sensibilité que les mots finissent par manquer pour le dire. Là où l'érotique traductologique à tendance pratique jouit de sa propre expérience, celle de son manque extatique, l'érotique traductologique à tendance théorique, pour sa part, se félicite d'avoir tout compris, ou du moins l'essentiel. On l'aura deviné, cette distinction recoupe la célèbre opposition platonicienne entre Eros et Agapè.

La caricature à laquelle se prête naturellement les extrêmes ne doit pas faire oublier que l'essentiel se joue dans la mise en relation. D.H. Lawrence, mais également Anaïs Nin et G. Bataille après lui, ont insisté sur la nécessité d'envisager « le fait sexuel que dans le cadre d'une totalité concrète et solidaire, où le monde érotique et l'intellectuel se complètent et se trouvent sur un plan d'égalité. » (Bataille 1970 : 19). En toute rigueur, il faudrait distinguer ce que ces trois projets de réconciliation - qui, répétons-le, valent également pour la traduction - ont chacun de singulier. L'érotique a ceci de commun avec la poétique de Meschonnic qu'elle se conçoit comme un projet global dont la traduction constitue une des dimensions. Plus précisément, l'érotique du traduire interroge autant le déni d'amour de la traductologie (son phallocentrisme, par exemple) que ses pratiques relationnelles. Dans cette perspective, la caresse ${ }^{6}$ porte la promesse d'une certaine mise en continuité entre le corps et l'esprit. En définitive, l'érotique du traduire offre un corps théorique et une sensibilité nouvelle à la traduction.

Partant, comment saisir l'activité érotique de la traduction dans sa dimension la plus relationnelle, la moins figée donc ? Parmi les nombreux gestes susceptibles de rendre compte de cette activité, la caresse nous paraît particulièrement pertinente. Déjà effleurée par J.P. Sartre dans l'Être et le Néant, cette notion occupe une position centrale dans la philosophie érotique d'Emmanuel Levinas. En outre, la caresse a fait l'objet de ré-élaborations par L. Irigaray et J-L. Marion qui, dans son Phénomène érotique, l'a affectée d'une sensualité inédite (Marion 2003 : 203). D'emblée, il convient de souligner le fait que l'érotique du traduire ne se saisit pas toute entière dans la caresse. Un certain nombre de gestes alternatifs sont possibles, à commencer par le «toucher» (die Berührung) fugace de W. Benjamin. Ces positions, autant qu'elles sont, pourraient être regroupées dans un recueil à venir qui tiendrait à la fois du Kama Sutra, des Théorèmes de la traduction de J.R. Ladmiral que de la Méthode du bien traduire d'Etienne Dolet. La perspective d'un tel ouvrage sera moins prescriptive qu'exploratoire : il s'agirait d'envisager la diversité des positions relationnelles (toucher, caresse) dont est capable la traduction.

De la caresse, on dira qu'elle met en échec la pornographie dans la mesure où elle ménage une part à l'invisible, à l'intraduisible sans pour autant les hypostasier. Ainsi, la traductioncaresse n'est-elle ni un geste d'appropriation, ni un geste de trahison. Dans un sens proche de celui de Levinas ${ }^{7}$, la traduction érotique déploie le texte original au-delà de son autorité en l'ouvrant à la perspective de l'Autre (langue). La caresse - autant que la « transgression » (Bataille) qui est un autre nom de la traduction - réalisent l'abandon du texte à lui-même et, ce faisant, la libération de ses potentialités interprétatives et traductives. Point capital, cet abandon n'est envisageable que dans la mesure où le traduire ne se fait pas contraignant, amène l'original à se perdre en toute confiance. Dès lors, il y n'a pas plus de raison pour célébrer cet « abus » de pouvoir qu'est la traduction (Nouss 2001) que de chercher à le rendre moins tragique, sinon 
moins douloureux. S'il n'y a certes pas d'amour heureux, comme le dit le poète, le malheur n'est pas une nécessité absolue ; il peut, à l'occasion, se tromper. Autrement dit, il n'y a pas de malheur totalement malheureux, cela serait trop beau. Entre la chimère de la traduction parfaite et l'éloge de la trahison, il conviendrait de faire la place à une pédagogie de l'abandon. Par là, nous entendons la mise en dialogue préliminaire entre la perte d'autorité de l'original (sur lui-même) et sa saisie en traduction (du point de vue de l'autre langue). C'est précisément à cette « croisée » (Marion 2003) que se tient le plaisir (éventuellement nostalgique) de la perte. En termes phénoménologiques, la pédagogie de l'érotique, c'est la traduction donnant, sans le forcer, l'abandon ou la trahison (à elle-même comme à l'autre) de l'original. Pour le dire autrement, l'abandon n'est pas nécessairement tragique : il s'obtient à force de caresses et de précautions. L'érotique du traduire est l'épreuve - la pédagogie et la pragmatique, si l'on préfère - de cet abandon.

Par ailleurs, l'érotique du traduire entend relancer le questionnement sur le corps du traducteur. Ainsi, les érotiques traductologiques ont-elles, chacune sur leur propre registre, affecté une corporéité au praticien. Pour les plus platoniques, ce corps s'abstrait tout entier dans la conscience du traducteur. Pour les plus (f)rigides, il n'y a de corps plus aimable que celui de l'original. L'exigence de fidélité finit par priver le corps du traducteur de sa ductilité, ne lui laissant, en tout et pour tout, qu'un manque d'amour dont l'invisibilité est l'expression la plus manifeste. Stigmate de son imperfection, le corps du traducteur est tellement rigide qu'il peine à cicatriser, ne sent même plus les coups. Si la traduction pornographique réduit le corps du traducteur à sa seule activité de performance, les traductologies scientifiques en font une machine lorsqu'elles n'accordent pas une importance démesurée à ses yeux et à ses mouvements de nuque. A la suite des réflexions de D. Robinson, l'érotique du traduire entend non seulement redonner sa place au « somatique », mais déployer ce corps traductif dans la pluralité de ses dimensions sensibles (charnelle, sensuelle, intellectuelle, intuitive, etc.).

\section{Vers un changement de paradigme ?}

Dans la conjoncture actuelle, le corps théorique de la traductologie semble partagé entre deux paradigmes dominants. D'une part, un paradigme éthique, d'inspiration bermanienne, qui revendique la figure transcendante d'une l'altérité envers laquelle le traducteur se voit obligé. D'autre part, un paradigme politique, dont le principal porte-drapeau se nomme L. Venuti, qui envisage le champ de la traduction en termes de rapports de force. En pratique, ces deux tendances se croisent : toute accueillante qu'elle est, l'éthique bermanienne ne cesse de se poser en termes d' « espaces ». A force de mettre l'emphase sur l'autre, une certaine exégèse finit par le prendre trop vite pour acquis. Rappelons à ceux-là que l'accueil bermanien de l'Autre ne va pas sans une double sublimation de la tendance ethnocentrique et de la haine de sa langue maternelle. Non seulement l'Autre est-il l'enjeu d'une lutte intérieure, mais il doit être débusqué dans le territoire intime de la langue maternelle. Acte éminemment stratégique, la traduction consiste, selon Berman, à « chercher-et-trouver le non-normé de la langue maternelle pour y introduire la langue étrangère et son dire. » (Berman 1999 : 131).

Toute conflictuelle qu'elle est, la politique de la traduction de L. Venuti s'efforce de faire une place à l'étranger et même à l'utopie. Cette utopie, c'est autant celle de l'obtention d'un consensus entre les cultures cible et source que l'espoir d'une possibilité d'accueil de l'étranger tel que la réalise la «foreignization » ou la pratique de l'interprète communautaire (Venuti 2000 : 486-488). En fait, l'utopie de L. Venuti est d'abord un projet politique de traduction qui, à ce titre, ne peut manquer d'être intéressé.

Partant de ce constat, l'érotique de la traduction pose les questions suivantes.

Premièrement, comment ramener l'abstraction éthique de l'Autre dans le corps du langage ? Il est ici davantage question d'épaisseur signifiante (musicalité, rythme, jeu de langage, etc.) que de littéralisme, si l'on entend par là l'impératif de la lettre. C'est, le croyons-nous, dans un certain écart contrôlé par rapport à elle-même - Bataille parlerait de transgression - que la lettre découvre son corps et peut en jouer. Deuxièmement, pourquoi ne pas envisager également les rapports de force sous l'angle complémentaire des relations amoureuses. À mille lieux de tout idéalisme niais, la caresse traduisante déploierait, sur le registre qui est le sien, une double activité éthique et politique. D'une part, elle se donnerait comme œuvre de charité ${ }^{8}$ dans les acceptions complémentaires de mise en abandon de l'original (que l'on distingue ici du motif classique de la trahison) et de traduction rien que pour le geste (que l'on oppose ici à la 
traduction comme appropriation). D'autre part, la caresse de traduction s'imposerait comme force de contestation face à l'ordre pornographique du traduire. Plus exactement, la traductioncaresse serait mise en échec du totalitarisme du visible et condition de réaménagement de l'invisibilité du traducteur.

\section{NOTES}

1. Il faudra bien distinguer les érotiques traductologiques (ou érotiques restreintes du traduire) de l'érotique du traduire au sens où nous l'entendons dans le présent article. Tandis que les premières malmènent, d'une façon ou d'une autre, le corps du texte, la seconde se défend, pour des raisons qui tiennent essentiellement à sa nature caressante, de tels abus. Dans les termes d'A. Berman, l'érotique du traduire est l'analytique des érotiques traductologiques, ce qui veut dire qu'elle en examine les tendances déformantes, c'est-à-dire les violences ou les masquages imposées au corps du texte.

2. Nous faisons bien évidemment allusion aux études sur l'évaluation de la pratique du traducteur sur la base de son activité visuelle au moyen d'un équipement informatique connu sous le nom d'Eye Tracking System. Selon l'érotique, il y a moins à répudier ce genre d'analyse (au nom d'un humanisme d'arrière-garde, par exemple), qu'à comprendre quel type de corporéité elle affecte au traducteur. À l'évidence, les nouvelles technologies (internet, dictionnaires en lignes, traitements de texte, TAO, etc.) se sont imposées au traducteur au point de devenir partie intégrante de sa personne et de son quotidien. Le fantasme de la machine de traduction a désormais fait place à la réalité, plus nuancée, du « traducteur-cyborg » (Cronin 2003 : 116-117).

3. L'érotique et le pornographique sont des catégories dont la morale se sert pour justifier, avec plus ou moins de raison, son arbitraire, c'est-à-dire aussi bien son ordre moral que son ouverture d'esprit.

4. L'érotique du traduire s'érige contre l'assimilation bourgeoise entre érotique et beau style. En ce sens,

l'érotique est peut-être avant tout une œuvre de contestation.

5. À ce niveau d'abstraction, le chiasme perd énormément en nuance et en sensibilité.

6. C'est particulièrement le cas dans l'écriture d'A. Nin où le motif omniprésent de la caresse opère comme un geste pragmatique d'écriture, appelant comme telle une traduction érotique au sens où nous l'entendons.

7. Dans le cadre de cet article, notre propos est davantage de proposer une définition générale de la traductioncaresse que de la rattacher à un cadre théorique particulier, celui de Levinas, par exemple.

8. Remarquons, au passage, que caresse et charité proviennent de la même racine latine : carus.

\section{RÉFÉRENCES}

BARTHES, R. (2000) : Le plaisir du texte, Paris, Éditions du Seuil.

Bataille, G. (1970) : « Histoire de l'érotisme », in Euvres complètes, t. VII, Paris, Gallimard.

BERMAN, A. (1984) : L'épreuve de l'Etranger, Paris, Gallimard.

Berman, A. (1995) : Pour une critique des traductions : John Donne, Paris, Gallimard.

BERMAN, A. (1999) : La traduction et la lettre ou l'auberge du lointain, Paris, Éditions du Seuil.

CRONIN, M. (2003) : Translation and Globalization, Londres/New York, Routledge.

Huston, N. (1982) : Mosaïque de la pornographie. Marie-Thérèse et les autres, Paris, Éditions

Denoël/Gonthier.

IRIGARAY, L. (1984) : «Fécondité de la caresse », in Ethique de la différence sexuelle, Paris, Éditions de Minuit.

LARBAUD, V. (1946) : De la traduction. Paris, Actes Sud.

LAWRENCE, D. H. (1993) : L'Amant de lady Chatterley (traduit de l'anglais par F. Roger-Cornaz), Paris, Folio classique.

LeVinAS, E. (1974) : Totalité et infini : essai sur l'extériorité, La Haye, M. Nijhoff.

LEVINAS, E. (1979) : Le temps et l'autre, Paris, Fata Morgana.

MARION, J.-L. (2003) : Le Phénomène érotique, Paris, Grasset.

MARZANo, M. (2003) : La pornographie ou l'épuisement du désir, Paris, Buchet Chastel.

Meschonic, H. (1999) : Poétique du traduire, Paris, Verdier.

Nouss, A. (2001) : «Éloge de la trahison », in Traduction, Terminologie, Rédaction 14-2, p. 167-181.

RAO, S. (2005) : « Towards an Erotics of Translation », in Actes du colloque "Tasking the Translator: on the

Practice and Theory of Translation in/for our Times », Cornell, Cornell University Press, à paraître.

RoBinson, D. (1992) : The Translator's turn, Baltimore, John Hopkins University Press.

SteineR, G. (1998) : Après Babel (traduit de l'anglais par L. Lotringer \& P.E Dauzat), Paris, Albin Michel. 
VeNuTI, L. (1992) : The Scandals of Translation, London/New York, Routledge.

Venuti, L. (1995) : The Translator's Invisibility, Londres/New York, Routledge.

Venuti, L. (2000) : «Translation, Community, Utopia », in Venuti, L. (ed.), The Translation Studies Reader, Londres/New York, Routledge, p. 468-488.

Von Flotow, L. (1997) : Translation and Gender. Translating in the Era of Feminism, Manchester, St Jerome - University of Ottawa Press. 